\title{
An Adaptive Communication Mechanism for Highly Mobile Agents
}

\author{
JinHo Ahn \\ Dept. of Computer Science, Kyonggi University \\ San 94-6 Yiuidong, Paldalgu, Suwonsi Kyonggido 442-760, Republic of Korea \\ jhahn@kyonggi.ac.kr
}

\begin{abstract}
Agent mobility causes reliable inter-agent communications to be more difficult to achieve in distributed agent based systems. To solve this issue, three representative agent tracking and message delivery mechanisms, broadcast-based, home-based and forwarding pointerbased, were previously proposed. However, due to their respective drawbacks, none of them is suitable for efficient delivery of messages to highly mobile agents, which move frequently between service nodes. This paper introduces an adaptive forwarding pointer-based agent tracking and message delivery mechanism to alleviate their disadvantages. The proposed mechanism allows each mobile agent to autonomously leave tails of forwarding pointers on some few of its visiting nodes depending on its preferences. Thus, it is more efficient in terms of message forwarding and location management than the previous forwarding pointer-based one. Simultaneously, it considerably reduces the dependency on the home node in agent location updating and message delivery compared with the home-based mechanism.
\end{abstract}

\section{Introduction}

Mobile agent is an autonomously running program, including both code and state, that travels from one node to another over a network carrying out a task on user's behalf [4]. Due to its beneficial characteristics, i.e., dynamicity, asynchronicity and autonomy, it has been primarily used as an enabling programming paradigm for developing distributed computing infrastructures in various application fields such as e-commerce, telecommunication, ubiquitous computing, active networks and the like 24. However, as the size of these fields is rapidly increasing, several research issues related to the mobile agent technology such as communication, security, dynamic adaptation, etc., should be reconsidered to be suitable for their scale. Among them, it is most important to enhance the performance of the agent communication in Internet-scale infrastructures. For this purpose, some effective and efficient inter-agent communication mechanism is required in distributed agent-based systems. Agent mobility may lead to the loss of messages being destined to an agent on its migration. Thus, it causes reliable inter-agent communications to be not easy to achieve in the distributed agent based systems. Especially, guaranteeing the delivery of messages 
to highly mobile agents, which move frequently among service nodes, is a more challenging problem, which this paper attempts to address. To consider the agent mobility, three representative agent tracking and message delivery mechanisms, broadcast-based, home-based and forwarding pointer-based, were previously proposed. The broadcast-based mechanism[7] guarantees transparent and reliable inter-agent communication and can also provide multicast communication to a set of agents. But, to locate the message destination, the mechanism has to contact every visiting node in the network. Thus, its large traffic overhead makes broadcasts impractical in large-scale distributed agent systems.

The home-based mechanism [5] is borrowed from the idea of Mobile IP [8]. It requires that each mobile node has a home node, and forces the mobile node to register its current temporary address, called care-of-address, with its home node whenever it moves. Thus, when some messages are sent to a mobile node currently located at a foreign network, the messages are first directed to its home node, which forwards them to the mobile one. This mechanism is simple to implement and results in little mobile node locating overhead. However, it is unsuitable for highly mobile agents in distributed agent based systems because every agent location updating and message delivery are all performed around the home agent, which introduces centralization. Moreover, the Mobile IP generally assumes each mobile node's home node is a static one whereas distributed agent based systems don't have this assumption, i.e., the home node may be disconnected from the network. Thus, this mechanism cannot address the disconnection problem.

In the forwarding pointer-based mechanism 36], each node on a mobile agent's movement path keeps a forwarding pointer to the next node on the path. Thus, if a message is delivered to an agent not being at the home node, the message must traverse a list of forwarding nodes. Thus, this mechanism can avoid performance bottlenecks of the global infrastructure, and therefore improve its scalability, particularly in large-scale distributed agent-based systems, compared with the home based one. Additionally, even if a home node is disconnected from the rest of the network, the forwarding pointer based mechanism allows agents registering with the node to communicate with other agents. However, as highly mobile agents leads to the length of their chains of pointers being rapidly increasing, its message forwarding overhead may be significantly larger. Furthermore, the number of forwarding pointers each service node needs to keep on its storage may exponentially increase if a large number of mobile agents are running in the systems. In a previous work[ [6], a type of update message called in form message was introduced to include an agent's current location for shortening the length of trails of forwarding pointers. In this case, a node that receives the message is allowed to update its table if the received information is more recent than the one it had. However, it introduces no concrete and efficient solutions for this purpose, for example, when update messages should be sent, and which node they should be sent to.

Therefore, we observe these respective drawbacks of the three previous mechanisms may be critical obstacles to efficient communications between highly mo- 
bile agents in large-scale distributed agent systems. This paper introduces an adaptive forwarding pointer-based agent tracking and message delivery mechanism to avoid their disadvantages. The proposed mechanism allows each mobile agent to autonomously leave trails of forwarding pointers only on some few of its visiting nodes depending on its preferences such as location updating and message delivery costs, security, network latency and topology, communication patterns, etc.. Thus, it is more efficient in terms of message delivery and location management than the previous forwarding pointer-based one. Additionally, it considerably decentralizes the role of the home node in agent location updating and message delivery. This feature alleviates the two problems of the home-based mechanism.

Due to space limitation, our system model, formal descriptions and correctness proof of the proposed mechanism, and related work are all omitted. The interested reader can find them in [1].

\section{The Adaptive Communication Mechanism}

As mentioned in section 1, the proposed adaptive communication mechanism is designed to have the following features unlike previous ones.

- Require small size of storage for location management per service node.

- Result in low message forwarding overhead.

- Reduce considerably the dependency on home node in agent location updating and message delivery.

First of all, let us define two important terms, forwarder and locator. Forwarder of an agent means a service node keeping a forwarding pointer of the agent on its storage. Thus, depending on the behavior of agent communication mechanisms, there may exist various number of forwarders of each agent in the system. Locator of an agent is the forwarder managing the identifier of the node where the agent is currently located. In this paper, it is assumed that there is only one locator in the system. To satisfy all the three requirements, our adaptive mechanism forces only some among all visiting nodes of each agent to be forwarders. This behavior can considerably reduce both the amount of agent location information each node needs to maintain and the delivery time of each message because the length of its forwarding path may be much more shortened. Also, since there exist multiple forwarders, not only one, in this mechanism, the home node centralization can be avoided. But, as a part of the vising nodes are forwarders in this mechanism, a new method is required to consistently manage location of each agent and enable each sent message to be delivered to the agent despite its migrations unlike in the previous forwarding pointer-based one. In the following, two components of the proposed mechanism, agent location management and message delivery algorithms, are explained in detail respectively.

Agent Location Management. For the agent location management algorithm, every node $N_{i}$ should maintain the following data structures. 
- RunningAgents: A table for saving location information of every agent currently running on $N_{i}$. Its element is a tuple (agent_id, locmngr_n, agent_t). locmngr_n is the identifier of agent agent_id's locator. agent_t is the timestamp associated with agent agent_id when the agent is located at $N_{i}$. Its value is incremented by one every time the corresponding agent migrates. Thus, when agent agent_id migrates to $N_{i}, N_{i}$ should inform locmngr_n of both its identifier and agent_t of the agent so that locmngr_n can locate the agent.

- AgentLocs ${ }_{i}$ : A table for saving location information of every mobile agent which is not currently running on $N_{i}$, but of which $N_{i}$ is a forwarder. Its element is a tuple (agent_id, destination_n, agent_t, ismanaging_f, ismigrating_f). destination_ $n$ is the identifier of the node where $N_{i}$ knows agent agent_id is currently located and running. agent_t is the timestamp associated with the agent when the agent is located at node $N_{\text {destination_n }}$. It is used for avoiding updating recent location information by older information [6]. ismanaging $f$ is a bit flag indicating whether $N_{i}$ is agent agent_id's locator or not. In the first case, its value is true and otherwise, false. ismigrating $f$ is a bit flag designating if the agent is currently migrating to another node $(=$ true $)$ or not $(=$ false $)$.

The algorithm for managing each agent's location on its migration is informally described using figure 1] This figure shows message interactions between nodes occurring in $a$ 's location updating and location information maintained by each node while migrating from its home node to $N_{1}$ through $N_{5}$. In figure 1(a), $a$ is created on $N_{\text {home }}$ and then an element for $a\left(i d_{a}\right.$, home, 0$)$ is saved into RunningAgents some in the first step. If a attempts to move to $N_{1}$ after having performed its partial task, in the second step, it inserts into AgentLocs home $a$ 's element $\left(i d_{a}, 1,1\right.$, true, true) indicating $N_{\text {home }}$ is $a$ 's locator and $a$ is currently moving to $N_{1}$. Then, $N_{\text {home }}$ dispatchs the agent with the identifier of the node and a's timestamp to $N_{1}$. When receiving these, $N_{1}$ increments the timestamp by one. In this case, as $a$ wants $N_{1}$ to be its locator, it inserts a's location information $\left(i d_{a}, 1,1\right)$ into RunningAgents $s_{1}$ in the third step. At the same time, $N_{1}$ sends $N_{\text {home }}$ a message changelmngr including a's timestamp in order to inform $N_{\text {home }}$ that $N_{1}$ is a's locator from now. On receiving the message, $N_{\text {home }}$ updates a's location information on AgentLocshome using the message and sets two fields of $a$ 's element, ismanaging $f$ and ismigrating $f$, all to false. If the messages destined to a have been buffered in $N_{\text {home's }}$ message queue due to the migration, they are transmitted to $N_{1}$. When $a$ attempts to migrate to $N_{2}$ after $a$ has performed a part of its task in the forth step, $N_{1}$ puts $a$ 's element $\left(i d_{a}, 2,2\right.$, true, true) into AgentLocs ${ }_{1}$ and then dispatchs agent $a$ to $N_{2}$. In this case, $N_{2}$ increments $a$ 's timestamp by one and then inserts $a$ 's element $\left(i d_{a}, 1,2\right)$ into Running Agents $s_{2}$ like in the fifth step because $a$ wants $N_{2}$ to be just a visiting node. Also, the node registers $a$ 's current location with its locator $N_{1}$ by sending a message update including the timestamp to $N_{1}$. If there are any messages sent to the agent in the queue of $N_{1}$, they are forwarded to $N_{2}$.

Figure 1(b) illustrates that agent a moves from $N_{2}$ to $N_{3}$. In this example, $N_{2}$ first sends $N_{1}$ a message $m_{-}$initiated indicating that $a$ 's migration process 
begins from now. When receiving the message, $N_{1}$ sets one field of a's element ismigrating_f to true in the second step and then send a message m_reply to $N_{2}$. Suppose the migration is started without the execution of this invalidation procedure. If $N_{1}$ receives any message destined to $a$ in this case, it forwards the message to $N_{2}$ because it doesn't know whether the migration continues to be executed. But, neither a may be currently running on $N_{2}$ nor $N_{2}$ keep a's location information on AgentLocs 2 because $N_{2}$ isn't a's forwarder. In this case, the message cannot be delivered to $a$. Therefore, $N_{2}$ has to push $a$ to $N_{3}$ after having received the message $m_{-} r e p l y$ from $a$ 's locator, and then remove $a$ 's element from Running Agents $s_{2}$. Afterwards, $a$ 's visiting node $N_{3}$ increments $a$ 's timestamp and saves $a$ 's element $\left(i d_{a}, 1,3\right)$ into RunningAgents $s_{3}$ in the third step, and then sends a message update to $N_{1}$. On the receipt of the message, $N_{1}$ updates $a$ 's element in AgentLocs ${ }_{1}$ to $\left(i d_{a}, 3,3\right.$, true, false) using the message.

Figure 11(c) shows an example that $N_{5}$ becomes $a$ 's locator when agent $a$ moves from $N_{4}$ to $N_{5}$. In this case, after $N_{5}$ creates a's location information $\left(i d_{a}, 5,5\right)$ and inserts it into RunningAgents $s_{5}$ in the third step, the node sends $N_{1}$ a message changelmngr for notifying the previous locator $N_{1}$ that $N_{5}$ is $a$ 's locator from now. Also, $a$ attempts to register its current location with $N_{\text {home }}$ in order to reduce the message delivery time incurred when another agent initially sends a message to $a$ via $N_{\text {home }}$. For this purpose, $N_{5}$ sends a message update to $N_{\text {home }}$. If a recognizes this consideration helps no performance improvement, it doesn't perform the home node update procedure. After that, $N_{\text {home }}$ and $N_{1}$ update $a$ 's location information respectively like in the third step.

Message Delivery. For the message delivery algorithm, every node $N_{i}$ should contain an agent location cache, ALocsCache , $_{i}$ as follows.

- ALocsCache ${ }_{i}$ : A cache for temporarily storing location information of each mobile agent which agents running on $N_{i}$ communicate with. Its element is a tuple (agent_id, forward_n, agent_t). forward_n is the identifier of the node where $N_{i}$ knows agent agent_id is currently located and running. Thus, when attempting to deliver messages to agent agent_id, each agent on $N_{i}$ forwards them to forward_n regardless of whether this address is outdated. agent_t is the timestamp assigned to agent agent_id when the agent was located at node $N_{\text {forward_n }}$.

We intend to use an example in figure 2 to clarify the algorithm to enable every sent message to be reliably delivered to its target agent despite agent migrations. This example illustrates agent $b$ sends three messages, $m s g 1, m s g 2$ and $m s g 3$ to agent $a$ in this order while $a$ is migrating from its home node to $N_{1}$ through $N_{6}$ according to its itinerary. In figure 2(a), after $a$ has moved from $N_{\text {home }}$ to $N_{2}, b$ at $N_{\text {sender }}$ will deliver the first message $m s g 1$ to $a$. In this case, $N_{\text {sender }}$ has no location information for $a$ in its location cache ALocsCache sender $_{\text {. Thus, }} N_{\text {sender }}$ creates and saves $a$ 's element $\left(i d_{a}\right.$, home, 0$)$

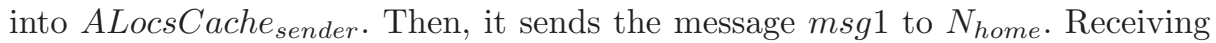
the message, $N_{\text {home }}$ retrieves a's element from AgentLocs home. In this case, as the value of the bit flag ismanaging in the element is false, $N_{\text {home }}$ isn't a's 

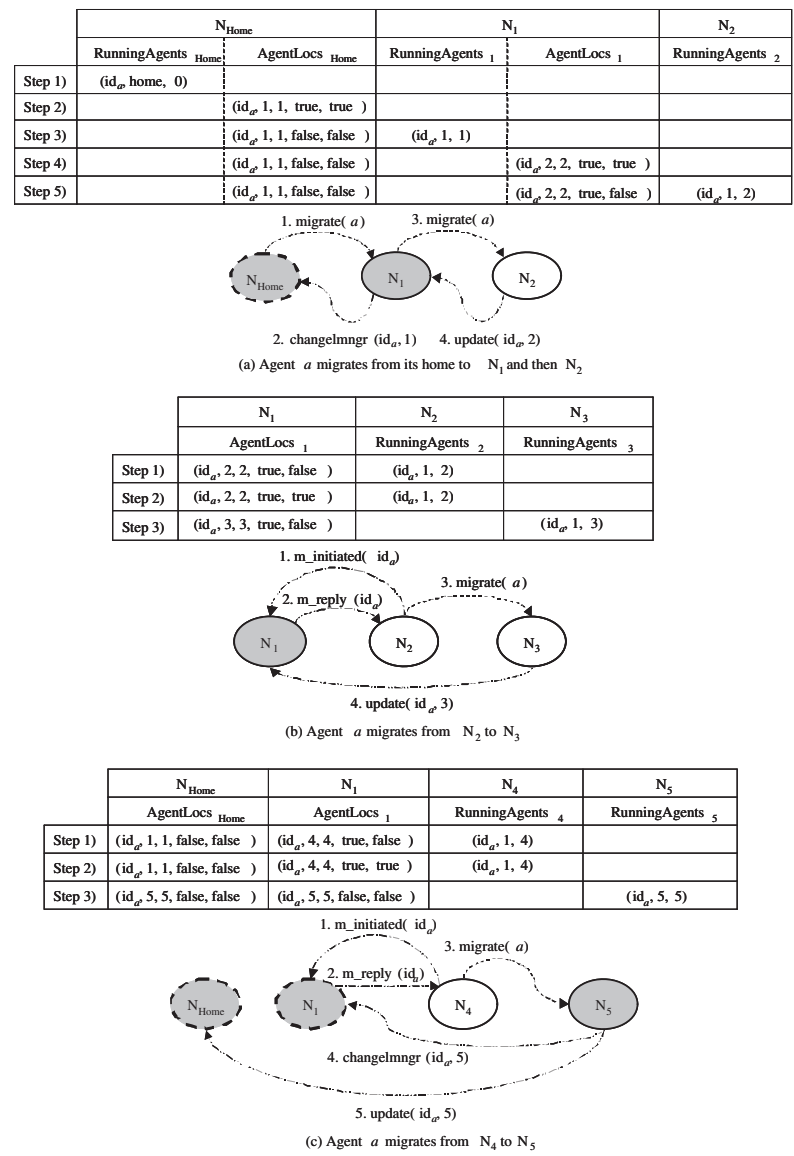

Fig. 1. An example of agent $a$ 's location updating on its migration according to its itinerary

locator. Thus, it consults the element and forwards the message $m s g 1$ to the next forwarder $N_{1}$. On the receipt of the message, $N_{1}$ obtains $a$ 's element from AgentLocs $_{1}$ and then checks the flag ismanaging in the element. In this case, $N_{1}$ is $a$ 's locator because the value of the flag is true. Also, as the value of the second flag ismigrating is false, it forwards the message to a's currently running node $N_{2}$ by consulting the element. At the same time, as $N_{\text {sender }}$ has the outdated identifier of a's locator, $N_{1}$ sends $N_{\text {sender }}$ a message updateCache containing the identifier of $a$ 's current $\operatorname{locator}\left(=N_{1}\right)$ and $\operatorname{timestamp}(=2)$ in figure 2(a). When receiving the message, $N_{\text {sender }}$ updates $a$ 's element in

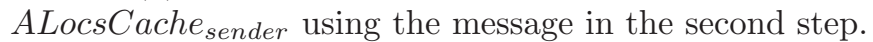

Figure 2(b) shows that $a$ migrates from $N_{2}$ to $N_{4}$ and then $b$ at $N_{\text {sender }}$ sends the second message $m s g 2$ to $a$. In this case, $N_{\text {sender }}$ finds $a$ 's element from ALocsCache sender and then forwards $m s g 2$ to $N_{1}$. On the receipt of 
$m s g 2, N_{1}$ can see that it is $a$ 's current locator because the value of the bit flag ismanaging of a's element in AgentLocs ${ }_{1}$ is true. Also, as the value of the flag ismigrating is false, $N_{1}$ can sends $m s g 2$ to $a$ 's currently running node $\left(=N_{4}\right)$. At this time, as $b$ knows the identifier of $a$ 's current locator, $N_{1}$ doesn't sends any message updateCache to $b$.

Figure 2(c) illustrates that after $a$ has migrated from $N_{4}$ via the next

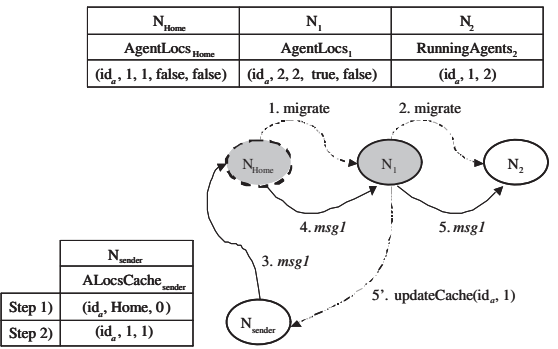

(a) Agent $b$ at host $\mathrm{N}_{\text {ouder }}$ sends message $m s g l$ to agent $a$ at $\mathrm{N}_{2}$ via $a$ 's home

\begin{tabular}{|c|c|c|c|}
\cline { 2 - 4 } \multicolumn{1}{c|}{} & $\mathrm{N}_{1}$ & $\mathrm{~N}_{3}$ & $\mathrm{~N}_{4}$ \\
\cline { 2 - 4 } \multicolumn{1}{c|}{} & AgentLocs ${ }_{1}$ & RunningAgents $_{2}$ & RunningAgents $_{3}$ \\
\hline Step 1) & $\left(\right.$ id $_{a}, 3,3$, true, false $)$ & $\left(\right.$ id $\left._{a}, 1,3\right)$ & \\
\hline Step 2) & $\left(\right.$ id $_{a}, 3,3$, true, true $)$ & $\left(\right.$ id $\left._{a}, 1,3\right)$ & \\
\hline Step 3) & $\left(\right.$ id $_{a}, 4,4$, true, false $)$ & & $\left(\right.$ id $\left._{a}, 1,4\right)$ \\
\hline
\end{tabular}

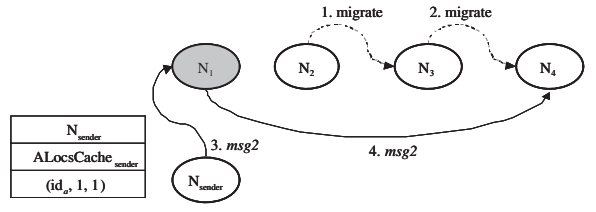

(b) Agent $b$ at $\mathrm{N}_{\text {seader }}$ sends message $m s g 2$ to agent $a$ at $\mathrm{N}_{4}$ via $a$ 's locator $\mathrm{N}_{1}$

\begin{tabular}{|c|c|c|c|c|}
\hline & $\mathrm{N}_{1}$ & \multicolumn{2}{|c|}{$\mathrm{N}_{5}$} & $\mathrm{~N}_{6}$ \\
\hline & AgentLocs, & RunningAgents $_{5}$ & AgentLocs $_{5}$ & RunningAgents 6 \\
\hline Step 1) & $\left(\mathrm{id}_{a}, 5,5\right.$, false, false $)$ & $\left(\mathrm{id}_{a^{\prime}}, 5,5\right)$ & & \\
\hline Step 2) & $\left(\mathrm{id}_{a}, 5,5\right.$, false, false $)$ & & $\left(\mathrm{id}_{a}, 6,6\right.$, true, true $)$ & \\
\hline Step 3) & $\left(\right.$ id $_{a}, 5,5$, false, false ) & & $\left(\mathrm{id}_{a}, 6,6\right.$, true, false $)$ & $\left(\mathrm{id}_{a}, 5,6\right)$ \\
\hline
\end{tabular}

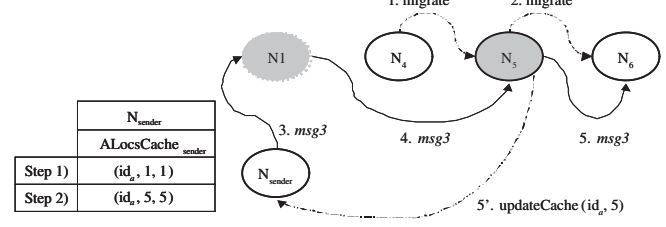

(c) Agent $b$ at $\mathrm{N}_{\text {snder }}$ sends message $m s g 3$ to agent $a$ at $\mathrm{N}_{6}$ via $a$ 's forwarder $\mathrm{N}_{1}$

Fig. 2. An example of agent $b$ sending three messages $m s g 1, m s g 2$ and $m s g 3$ to agent $a$ in order while $a$ migrating to several nodes according to its itinerary

locator $N_{5}$ to $N_{6}, b$ transmits the third message $m s g 3$ to $a$. In this figure, when the migration has been completed, three nodes $N_{1}, N_{5}, N_{6}$ have each a's location information like in the step 3. First, $N_{\text {sender }}$ sends $m s g 3$ to $N_{1}$ by consulting a's element in ALocsCachesender. In this case, as the value of the flag ismanaging of a's element in AgentLocs 1 is false, $N_{1}$ forwards msg3 to 
the next forwarder $N_{5}$. When $N_{5}$ receives the message, it transmits the message to $N_{6}$ because it recognizes that it is $a$ 's locator and $a$ isn't currently migrating. Concurrently, $N_{5}$ informs $N_{\text {sender }}$ that $a$ 's current locator is $N_{5}$ by sending a message updateCache to $N_{\text {sender }}$.

\section{Conclusion}

In this paper, an adaptive agent communication mechanism was introduced to considerably reduce both the amount of agent location information maintained by each service node and the delivery time of each message while reducing the dependency on the home node. To achieve this goal, this mechanism forces only a small part of all visiting nodes of each agent to becomes forwarders. In this mechanism, each agent should register its location with its current locator on every migration until it arrives at the next locator of the agent. This method may result in slightly higher location update cost per agent migration compared with that of the previous forwarding pointer-based mechanism. However, if each agent determines some among its visiting nodes as forwarders by properly considering several performance factors, the gap between the two costs may be almost negligible. Moreover, the mechanism allows the identifier of each agent's locator to be kept on the agent location cache of a node. This behavior highly reduces the cache update frequency of the node compared with the previous mechanism.

Future work is focused on selecting appropriate forwarders of each agent according to the changes which the agent senses in its environment related to location updating and message delivery costs, security policies, network latency and topology, communication patterns.

\section{References}

1. J. Ahn. Adaptive Communication Mechanisms for Mobile Agents. Technical Report KGU-CS-03-050, Kyonggi University, 2003.

2. P. Bellavista, A. Corradi and C. Stefanelli. The Ubiquitous Provisioning of Internet Services to Portable Devices. IEEE Pervasive Computing, Vol. 1, No. 3, pp. 81-87, 2002.

3. J. Desbiens, M. Lavoie and F. Renaud. Communication and tracking infrastructure of a mobile agent system. In Proc. of the 31st Hawaii International Conference on System Sciences, Vol 7., pp. 54-63, 1998.

4. A. Fuggetta, G.P.Picco and G. Vigna. Understanding Code Mobility. IEEE Transactions on Software Engineering, Vol. 24, No. 5, pp. 342-361, 1998.

5. D. Lange and M. Oshima. Programming and Deploying Mobile Agents with Aglets. Addison-Wesley, 1998.

6. L. Moreau. Distributed Directory Service and Message Router for Mobile Agents. Science of Computer Programming, Vol. 39, No. 2-3, pp. 249-272, 2001.

7. A. L. Murphy and G. P. Picco. Reliable Communication for Highly Mobile Agents. Journal of Autonomous Agents and Multi-Agent Systems, Vol. 5, No. 1, pp. 81-100, 2002.

8. C. Perkins, IP Mobility Support. RFC 2002, October 1996. 